\title{
A cross-sectional study of the relationships between different components of sarcopenia and brachial ankle pulse wave velocity in community- dwelling elderly
}

\author{
Yu-Dong Rong ( $\nabla$ ydrong2001@163.com ) \\ Tianjin First Center Hospital \\ Ai-Lin Bian \\ Tianjin First Central Hospital \\ Hui-Ying $\mathrm{Hu}$ \\ Tianjin First Central Hospital \\ Yue Ma \\ Yanda International Hospital \\ Xin-Zi Zhou \\ Tianjin First Central Hospital
}

\section{Research article}

Keywords: Elderly, Sarcopenia, Arterial stiffness, Brachial ankle pulse wave velocity

Posted Date: February 19th, 2020

DOI: https://doi.org/10.21203/rs.2.15968/v2

License: (c) This work is licensed under a Creative Commons Attribution 4.0 International License. Read Full License

Version of Record: A version of this preprint was published at BMC Geriatrics on March 30th, 2020. See the published version at https://doi.org/10.1186/s12877-020-01525-8. 


\section{Abstract}

Background: Previous studies have just found skeletal muscle mass decline is associated with arterial stiffness, but it is unclear whether muscle strength and physical performance as important compositions of sarcopenia are associated with arterial stiffness. The aim of this study was to investigate the relationship between sarcopenia, the components of sarcopenia and arterial stiffness among elderly in the community.

Methods: This study enrolled 450 elderly people who received general medical examinations in Tianjin First Central Hospital. Each of the subjects was greater than 65 years old, including 266 male and 184 female subjects. Based on the diagnostic criteria for sarcopenia in older people developed by the Asian Working Group for Sarcopenia (AWGS), 89 subjects were separated into the sarcopenia group. The living habits, disease status, general status and laboratory examinations of all subjects were collected. The body composition (including appendicular skeletal muscle mass and visceral fat area (VFA) of each participant) was measured by bioimpedance analysis. HS, usual gait speed (GS), and brachial ankle pulse wave velocity (baPWV) were measured.

Results : Sarcopenia subjects had higher baPWV, nutrition risk and lower appendicular skeletal muscle index(ASMI), Handgrip strength(HS), GS, body mass index(BMI), triacylglycerol(TG), serum albumin(ALB) and creatinine $(\mathrm{Cr})$ than did non-sarcopenia subjects; Sarcopenia subjects also had higher visceral fat area (VFA) than did non-sarcopenia subjects $(p<0.05)$. ASMI and HS were negatively correlated with baPWV $(t=-5.807, p=0.000$ and $t=-3.085, p=0.002)$, but the relationship between baPWV and GS was not statistically significant $(t=-0.862, p=0.389)$ by multivariable linear regression. After adjusting for confounders, a multivariate logistic regression analysis revealed that sarcopenia was related with age, $\mathrm{BMI}$, sports and baPWV in community dwelling elderly.

Conclusions: ASMI and HS were negatively correlated with baPWV in community dwelling elderly in China; and baPWV was a risk factor of sarcopenia.

\section{Background}

Sarcopenia, a newly recognized geriatric syndrome, is characterized by age-related declines in skeletal muscle in addition to decreased muscle strength and/or physical performance [1]. As the elderly population continues to grow rapidly worldwide, chronic geriatric diseases have become a global social problem. Among these diseases, sarcopenia is easily neglected, yet it can cause many adverse outcomes in the elderly including increased risk of falls and fractures, prolonged time in bed, increased risk of infection, and increased rates of cardiovascular disease with a poor prognosis [2].

Atherosclerosis is a major disease that causes death and impairs the quality of life of countless elderly persons, in whom its onset can often go unnoticed. The pathogenesis of atherosclerosis is similar to that of sarcopenia in many ways, including low physical activity, increases in oxidative stress and insulin resistance, changes in aging-related hormones, and increased levels inflammatory factors. Therefore, 
there is believed to be a correlation between sarcopenia and atherosclerosis. Although several studies have found that skeletal muscle mass decline is associated with arterial stiffness[3,4], but it is unclear whether muscle strength and physical performance as important compositions of sarcopenia are associated with arterial stiffness. And they used different protocol to diagnose sarcopenia before the Consensus Report of the Asian Working Group in 2014[1].The present study was therefore designed to investigate the relationship between sarcopenia, the components of sarcopenia and arterial stiffness according to the Consensus Report of the Asian Working Group in 2014 in Chinese community dwelling elderly.

\section{Methods}

Research subjects

A total of 450 elderly subjects ( 266 male and 184 female) aged $65-90$ attended general medical examinations from May 2015 to September 2016 in the Health Management Centre of Tianjin First Central Hospital, China. 89 of these individuals were diagnosed as having sarcopenia according to the criteria of AWGS. These subjects included 50 males and 39 females between the ages of 65-90 (mean age $72.48 \pm 4.65$ years). The other remaining 361 individuals without sarcopenia including 216 males and 145 females between the ages of 65-89 (mean age 71.05 \pm 4.15 years) served as the non-sarcopenia group in this study.

Inclusion criteria

People over the age of 65 that had undergone a general medical examination and baPWV examination in the Health Management Centre of Tianjin First Central Hospital who could walk by themselves and who did not have a history of mental illness were selected for inclusion in this study. All subjects agreed to participate in the study, and signed informed consent forms.

Exclusion criteria

Subjects with cognitive impairment or dementia, neurological diseases (central nervous system and peripheral nervous system diseases such as serious cerebral stroke, brain and spinal cord tumors, trauma, inflammation, or Parkinson's disease), or mental disorders affecting activity; organ failure (heart, lung, liver, kidney); edema, severe endocrine diseases that not be well-controlled; autoimmune diseases; inflammatory disease; malignancy or anorexia; malabsorption or gastrointestinal disorders, and trauma or fracture leading to bed rest were excluded from this study.

\section{Diagnosis of sarcopenia}

Sarcopenia was defined according to the Consensus Report produced by AWGS[1], which is criterion1 and/or criterion 2 plus criterion3. Criterion 1 was low muscle strength: $\mathrm{HS}<26 \mathrm{~kg}$ for men and $<18 \mathrm{~kg}$ for women. Criterion2 was low physical performance: usual GS $<0.8 \mathrm{~m} / \mathrm{s}$; Criterion 3 was low muscle mass: ASMl $<7.0 \mathrm{~kg} / \mathrm{m}^{2}$ for men and $5.7 \mathrm{~kg} / \mathrm{m}^{2}$ for women as measured by bioimpedance analysis. 
Muscle strength and mass, and gait speed

HS was measured using a hand-muscle developer (WCS-II, Beijing). HS was assessed for each hand, and the maximum HS of the right or left hand was analyzed.

GS was determined based on a subject's usual walking pace over a distance of 6 meters in seconds, counted after a one meter initial distance. GS measurement was conducted in a flat and unobstructed clinic hallway.

ASMI was defined as appendicular skeletal muscle mass (ASM)/height $\left(\mathrm{m}^{2}\right)$. ASM and VFA was measured by bioelectrical impedance analysis (InBodyS10, InBody Japan Inc., Tokyo, Japan). Lean body mass as measured by bioelectrical impedance analysis was highly correlated with the data evaluated by dual-energy X-ray absorptiometry [5].

Medical history and living habits

Acquisition of medical history and living habits was conducted using a questionnaire survey that included questions pertaining to smoking habits, sports participation, and past medical history (including hypertension, diabetes, cardiac disease, respiratory and urinary system diseases, dementia, neurological and mental disorders, serious cerebral stroke, edema, severe endocrine diseases, autoimmune diseases, inflammatory disease, malignancy or anorexia and malabsorption or gastrointestinal disorders). Smoking was defined as people who have smoked for six consecutive months or six cumulative months. Sports participation was defined as $>30 \mathrm{~min}$ in a given session, $>3$ times a week and lasting for more than 3 months.

Body measurements

Measurements of height, weight, and blood pressure $(\mathrm{mmHg})$ were routinely collected, and BMI was calculated.

BaPWV measurements:

On the day of medical examination between 8:00-11:00 a.m., the baPWV was measured via VP1000 (an automatic atherosclerosis tester produced by the Colin Company of Japan). The room temperature was kept at $22^{\circ} \mathrm{C}-25^{\circ} \mathrm{C}$. Before measurement, the subjects were instructed not to smoke and to rest for more than 5 minutes. The subjects were kept quiet, lying on the bed with their hands on both sides of their body. Blood pressure cuffs were tied to the upper arms and lower limbs separatelys. The upper arm cuff was oriented towards the brachial artery and the lower edge of the cuff was $2-3 \mathrm{~cm}$ away from the elbow. The lower limb cuff was located at the preaxial portion of that lower limb. The lower edge of the cuff was 1$2 \mathrm{~cm}$ away from the medial malleolus. The heart sound acquisition device was placed in the anterior cardiac region of the subject. The left and right wrists were clamped using an electrocardiogram acquisition device. Measurements were repeated twice for each subject, with the second measurement 
acquired as the final result. In this study, the larger values of baPWV on the left and right sides were analyzed.

Nutrition risk

To evaluate the nutritional risk, subjects were administered the Mini-nutritional Assessment short-form (MNA-SF); a score of less than or equal to 11 points indicated a risk of malnutrition.

Laboratory examinations

$5 \mathrm{ml}$ of elbow venous blood was drawn in the morning from each subject following a minimum of an 8 hour fasting period. This sample was then use to aquire a complete blood cell count, and to measure hemoglobin $(\mathrm{Hb})$ and blood biochemistry indicators including serum total protein, serum ALB, TG, total cholesterol, low-density lipoprotein cholesterol (LDL-C), high density lipoprotein cholesterol, serum $\mathrm{Cr}$, urea nitrogen, blood glucose, and hemoglobin $\mathrm{A} 1 \mathrm{C}(\mathrm{HbA} 1 \mathrm{C})$ were tested immediately.

Statistical methods

All data were analyzed using Statistical software Package for Social Sciences version 19.0 (SPSS 19.0). Continuous variables were given as mean \pm standard deviation (SD). Numeration data were described as percentages (\%).The differences in means and proportions between the two groups were analyzed using independent samples $t$ tests and chi-squared tests respectively. Multiple linear regression analysis was used to analyze the relationship between baPWV and ASMI, muscle strength $₫$ physical performance. Multiple logistic regression analysis was performed to estimate the odds ratios (OR) and $95 \%$ confidence intervals $(\mathrm{Cl})$ for arterial stiffness in relation to sarcopenia. To understand whether potential confounders could affect OR, we used a multivariate model with adjustment for the following covariates: age, sex, BMI, VFA, hypertension, diabetes, cardiovascular disease, smoking behaviors, physical activity, nutritional risk, TG, LDL-C, HbA1C, Hb, ALB, Cr. A p value $<0.05$ was considered statistically significant.

\section{Results}

(1) The general characteristics and the other clinical or biochemical indicators of the participants are listed in Table 1. Subjects with sarcopenia had higher baPWV, nutrition risk and lower ASMI, HS, GS, BMI, TG, serum ALB, and serum $\mathrm{Cr}$ than those without sarcopenia. Subjects with sarcopenia also had a higher VFA than did the subjects without sarcopenia. These differences between the two groups were significant $(P<0.05)$.

(2) The results of a multivariable linear regression analysis between baPWV and ASMI『muscle strength physical performance are listed in Table 2. In order to analysis the relationship between baPWV and components of sarcopenia including ASMI $\llbracket$ muscle strength and physical performance, components were tested separately and peformed a final analysis. BaPWV was taken as the dependent variable, ASMI, HS and GS were tested simultaneously as independent variables and models were adjusted for sex, age, BMI, VFA, hypertension, diabetes, cardiac, smoking, sports participation, MNA-SF, TG, LDL-C, HbA1C, Hb, ALB 
and $\mathrm{Cr}$. ASMI and HS were negatively correlated with baPWV ( $t=-5.807, p=0.000$ and $t=-3.085, p=0.002)$, but the relationship between baPWV and GS was not statistically significant $(t=-0.862, p=0.389)$ by multivariable linear regression.

(3) The relationship between arterial stiffness and sarcopenia

Univariate analyses were performed investigating the relationship between sarcopenia and baPWV, the other clinical or biochemical parameters measured in this study. The result are presented in Table 3 . The associations were observed between sarcopenia and age, BMI, Sport, MNA-SF, VFA and baPWV. Finally, we performed multivariate logistic regression analyses to assess the relationship between sarcopenia and baPWV(Table 4). Following adjustment for potential confounders (age, BMI, sport, MNA-SF and VFA), sarcopenia was related with age, BMI, sports and baPWV, baPWV was a risk factor of sarcopenia(OR=1.68, 95\% Cl 1.45-1.87).

\section{Discussion}

The main findings of this cross-sectional study were that those with sarcopenia had higher baPWV and lower ASMI, HS and GS than did non-sarcopenia subjects. Subjects with sarcopenia also had higher VFA. Multivariable linear regression analysis revealed ASMI and HS were negatively correlated with baPWV, but there was no significient correlation between GS and baPWV. Whereas baPWV was a risk factor of sarcopenia.

The prevalence of sarcopenia in this study was $18.8 \%$ and $21.2 \%$ in men and women respectively. It is somehow lower than the report conducted in older hospitalized patients[6] using the diagnostic criteria of the European Working Group on Sarcopenia in Older People (EWGSOP)[7]. We investigated the relationship between sarcopenia and arterial stiffness in the elderly of the community in China based on the criteria included in the Consensus Report of the Asian Working Group for Sarcopenia. In the present study population, baPWV increased as skeletal muscle mass decreased $(t=-5.807, p=0.000)$. Consistent with the results of our study, several studies have previously reported the correlation between skeletal muscle mass decline and arterial stiffness. A cross sectional study conducted in Japan on healthy middle-aged to elderly men demonstrated that low thigh muscle cross-sectional area corrected by body weight was significantly negatively associated with baPWV and carotid intima-media thickness, even after correction for confounding factors including age, body height, and physical activity[3]. Another cross-sectional survey in Japan of 97 hospitalized elderly postmenopausal women with type 2 diabetes mellitus (average age $=65$ years) found that after adjusting for confounding factors such as age, body mass index, blood lipids, systolic blood pressure, and duration of onset of type 2 diabetes mellitus, baPWV increased as relative skeletal muscle mass index decreased [4]. In addition, studies have also shown that there is a correlation between body composition and arterial calcification in elderly men $[8,9]$.

These previous studies[3,4] have just found skeletal muscle mass decline is associated with arterial stiffness, but as important compositions of sarcopenia, muscle strength or physical performance were not involved. Only few previous studies $[10,11]$ estimated the association between muscle strength or 
physical performance and arterial stiffness. Objects of the few previous studies were not sarcopenia and there are no consistent conclusions. Watson et al.[10] reported that higher PWV was associated with slower gait speed independent of hypertension and other risk factors only among those with peripheral artery disease(PAD), but not in well functioning cohort. Gonzales reported gait performance was inversely correlated with carotid-femoral PWV and carotid artery stiffness index after adjustment for age, body mass index, waist circumference and systolic blood pressure[11]. HS are proved to be associated with allcause mortality and cardiovascular mortality [12,13]. Hirotomo Yamanashi et al reported arterial stiffness was associated with HS in non-hypertensive populations, but not in hypertensive populations [14]. Whereas, a long-term study result showed that there was no relation between HS and arterial stiffness[15]. The present cross-sectional study analysed the relation between all compositions of sarcopenia (including skeletal muscle mass, muscle strength or physical performance) and baPWV as marker of arterial stiffness simultaneously. We revealed ASMI and HS as composition of sarcopenia were negatively correlated with baPWV ( $t=-5.807, p=0.000$ and $t=-3.085, p=0.002)$, but not GS. The result might mean decreased physical performance may have no effect on atherosclerosis.

Conversely, arterial stiffness may also affect sarcopenia. Arterial stiffness induces the decreasing flow of blood, oxygen, and nutrients to muscle tissue, leading to a loss of muscle mass. The Health $A B C$ study, a large cohort study of elderly individuals, investigated the role of arterial stiffening in sarcopenia. The results showed that arterial stiffening as measured based on carotid-femoral pulse wave velocity was associated with a decline in skeletal muscle mass [16]. This present cross-sectional study revealed sarcopenia was related with advancing baPWV $(\mathrm{OR}=1.68,95 \% \mathrm{Cl} 1.45-1.87)$. We speculate that sarcopenia interacts with atherosclerosis.

The pathophysiological mechanism underlying the association between sarcopenia and atherosclerosis have not been fully identified. Timely screening people at risk for sarcopenia and taking effective measures may help control or delay the progress of sarcopenia and arterial stiffness. Defining blood biomarkers in sarcopenia may help characterize the mechanisms of sarcopenia, allowing for a personalized follow-up for the effectiveness of prevention and treatment measures[17].

There are some limitations to this present study: (1) The gold standard for evaluating atherosclerosis, carotid to femoral pulse wave velocity (cfPWV), was not used in this study. Even so, baPWV is well correlated with cfPWV[18], and the AHA had included baPWV in the recommended criteria for evaluating atherosclerosis. (2) The significant differences in the age ranges between sarcopenic and non-sarcopenic participants was a major limitation of this study. Even though age was considered as a possible confounder in the multivariate models, the two groups were non-homogeneous. That may result in deviations from reality. Age matching in future research will be important. (3) The cross-sectional design is another limitation of this study, as we cannot determine cause-effect associations between sarcopenia and atherosclerosis. Therefore, prospective studies are needed. (4) It has been found that polypharmacy was associated with sarcopenia[19]. We did not consider polypharmacy as a covariate in this study. (5) The relatively small sample size of our study is also a limitation. 


\section{Conclusions}

In summary, as important compositions of sarcopenia-ASMI and HS were negatively correlated with baPWV in community dwelling elderly in China; and baPWV was a risk factor of sarcopenia. We revealed the interactive relationship between sarcopenia, compositions of sarcopenia and arterial stiffness.

\section{Declarations}

\section{Ethics approval and consent to participate}

This study was conducted in accordance with the Declaration of Helsinki. This study was approved by the Ethics Committee of Tianjin First Central Hospital. We explained to all participants the purpose of the study and how the data collected in this research study would be used.

\section{Consent for publication}

All participants provided written and informed consent.

\section{Availability of data and materials}

The datasets generated and/or analyzed during the current study are not publicly available due to the need to protect patient privacy and the Fund provider requires data secrecy.

\section{Competing interests}

The authors declare that they have no competing interests.

\section{Funding}

This research was supported by the Science and Technology Fund of Tianjin city health and Family Planning Commission. The funding was used for the collection of data.

\section{Author contributions}

RYD and BAL made substantial contributions to conception and design; HHY, MY and ZXZ were involved in acquisition of data, analysis, and interpretation of data; RYD drafted the manuscript and revised the version to be published. All authors read and approved the final manuscript.

\section{Acknowledgements}

Not Applicable.

\section{Abbreviations}


AWGS: the Asian Working Group for Sarcopenia; HS: handgrip strength; GS: gait speed; BMI: body mass index; ALB: serum albumin; TG: triacylglycerol; Cr: creatinine; VFA: visceral fat tissue; baPWV: brachial ankle pulse wave velocity; MNA-SF: mini-nutritional assessment short-form; BIA: Bioelectrical impedance analysis; ASM: appendicular skeletal lean mass; ASMI: appendicular skeletal muscle index; $\mathrm{Hb}$ : hemoglobin; HbA1C:hemoglobin A1C; LDL-C: low-density lipoprotein cholesterol; SD: standard deviation; cfPWV: carotid to femoral pulse wave velocity; OR:odds ratios; Cl:confidence intervals; PAD: peripheral artery disease.

\section{References}

1.Chen LK, Liu LK, Woo J, Assantachai P, Auyeung TW, Bahyah KS, Chou MY, Chen LY, Hsu PS, Krairit O, Lee JS, Lee WJ, Lee Y, Liang CK, Limpawattana P, Lin CS, Peng LN, Satake S, Suzuki T, Won CW, Wu $\mathrm{CH}$, Wu SN, Zhang T, Zeng P, Akishita M, Arai H. Sarcopenia in Asia: consensus report of the Asian Working Group for Sarcopenia. J Am Med Dir Assoc, 2014; 15(2):95-101.

2.Walrand S, Guillet C, Salles J, Cano N, Boirie Y. Physiopathological mechanism of sarcopenia. Clin Geriatr Med. 2011;27(3):365-85.

3.Ochi M, Kohara K, Tabara Y, Kido T, Uetani E, Ochi N, Igase M, Miki T. Arterial stiffness is associated with low thigh muscle mass in middle-aged to elderly men. Atherosclerosis 2010;212(1):327-32.

4.Tanaka KI, Kanazawa I,Sugimoto T. Reduced muscle mass and accumulation of visceral fat are independently associated with increased arterial stiffness in postmenopausal women with type 2 diabetes mellitus. Diabetes Res Clin Pract,2016;122:141-147.

5.Ling CH, de Craen AJ, Slagboom PE, Gunn DA, Stokkel MP, Westendorp RG, Maier AB. Accuracy of direct segmental multi-frequency bioimpedance analysis in the assessment of total body and segmental body composition in middle-aged adult population. Clin Nutr. 2011;30(5):610-5.

6.Bianchi L, Abete P, Bellelli G, Bo M, Cherubini A, Corica F, Di Bari M, Maggio M, Manca GM, Rizzo MR, Rossi AP, Landi F, Volpato S; GLISTEN Group

Investigators.Prevalence and Clinical Correlates of Sarcopenia, Identified According

to the EWGSOP Definitionand Diagnostic Algorithm, in Hospitalized Older People: The GLISTEN Study.J Gerontol A Biol Sci Med Sci. 2017;72(11):1575-1581.

7.Cruz-Jentoft AJ, Baeyens JP, Bauer JM, Boirie Y, Cederholm T, Landi F, Martin FC, Michel JP, Rolland Y, Schneider SM, Topinková E, Vandewoude M, Zamboni M; European Working Group on Sarcopenia in Older People. Sarcopenia: European consensus on definition and diagnosis: report of the European Working Group on Sarcopenia in Older People. Age Ageing. 2010;39(4):412-23.

8.Alexandersen P, Tankó LB, Bagger YZ, Jespersen J, Skouby SO, Christiansen C. Associations between aortic calcification and components of body composition in elderly men. Obesity,2006;14(9):1571-8. 
9.Ko BJ, Chang Y凶Jung HS, Yun KE, Kim CW, Park HS, Chung EC, Shin H, Ryu S. Relationship between low relative muscle mass and coronary artery calcification in healthy adults. Arterioscler Thromb Vasc Biol, 2016;36(5): 1016-21.

10.Watson NL, Sutton-Tyrrell K, Youk AO, Boudreau RM, Mackey RH, Simonsick EM, Rosano C, Hardy SE, Windham BG, Harris TB, Najjar SS, Lakatta EG, Atkinson HH, Johnson KC, Bauer DC, Newman $A B$; Health $A B C$ Study. Arterial stiffness and gait speed in older adults with and without peripheral arterial disease. Am J Hypertens. 2011;24(1):90-5.

11.Gonzales, JU. Gait performance in relation to aortic pulse wave velocity, carotid artery elasticity and peripheral perfusion in healthy older adults. Clin Physiol Funct Imaging, 2013;33(4):245-51.

12.Garcia-Hermoso A, Cavero-Redondo I, Ramirez-Velez R, Ruiz JR, Ortega FB, Lee DC, Martinez-Vizcaino V. Muscular Strength as a Predictor of All-Cause Mortality in an Apparently Healthy Population: A Systematic Review and Meta-Analysis of Data From Approximately 2 Million Men and Women. Arch Phys Med Rehabil. 2018; 99 (10):2100-2113.

13.Celis-Morales CA, Welsh P, Lyall DM, Steell L, Petermann F, Anderson J, lliodromiti S, Sillars A, Graham N, Mackay DF, Pell JP, Gill JMR, Sattar N, Gray SR. Associations of grip strength with cardiovascular, respiratory, and cancer outcomes and all cause mortality: prospective cohort study of half a million UK Biobank participants. BMJ. 2018; 361: k1651.

14. Yamanashi H, Kulkarni B, Edwards T, Kinra S, Koyamatsu J, Nagayoshi M, Shimizu Y, Maeda T, Cox SE. Association between atherosclerosis and handgrip strength in non-hypertensive populations in India and Japan. Geriatr Gerontol Int 2018; 18(7): 1071-1078.

15.van Dijk SC, Swart KM, Ham AC, Enneman AW, van Wijngaarden JP, Feskens EJ, Geleijnse JM, de Jongh RT, Blom HJ, Dhonukshe-Rutten RA, de Groot LC, van Schoor NM, Lips P,Uitterlinden AG, Mattace Raso FU, Smulders YM, van den Meiracker AH, van der Velde N. Physical Fitness, Activity and Hand-Grip Strength Are Not Associated with Arterial Stiffness in Older Individuals. J Nutr Health Aging 2015;19 (7): 779-84.

16.Abbatecola AM, Chiodini P, Gallo C, Lakatta E, Sutton-Tyrrell K, Tylavsky FA, Goodpaster B, de Rekeneire N, Schwartz AV, Paolisso G, Harris T; Health ABCstudy. Pulse wave velocity is associated with muscle mass decline: Health ABC study. Age (Dordr)2012;34(2):469-78.

17. Curcio F, Ferro G, Basile C, Liguori I, Parrella P, Pirozzi F, Della-Morte D,Gargiulo G, Testa G, Tocchetti CG, Bonaduce D, Abete P. Biomarkers in sarcopenia: A multifactorial approach.Exp Gerontol. 2016;85:1-8.

18. Tanaka H, Munakata M, Kawano Y, Ohishi M, Shoji T, Sugawara J, Tomiyama H, Yamashina A, Yasuda H, Sawayama T, Ozawa T. Comparison between carotid-femoral and brachial-ankle pulse wave velocity as measures of arterial stiffness. J Hypertens, 2009;27(10):2022-7. 
19. Hao Q, Hu X, Xie L, Chen J, Jiang J, Dong B, Yang M. Prevalence of sarcopenia and associated factors in hospitalised older patients: A cross-sectional study. Australas J Ageing. 2018;37(1):62-67.

\section{Tables}

Table 1 The general characteristics and clinical data of subjects with and without sarcopenia

\begin{tabular}{lllll}
\hline Variable & Sarcopenia & Non-Sarcopenia $\mathrm{n}=361$ & /t & $\mathrm{p}$ \\
& $\mathrm{n}=89$ & & & \\
\hline Sex $(\mathrm{male} / \mathrm{female})$ & $50 / 39$ & $216 / 145$ & 0.394 & 0.530 \\
\hline Age $(\mathrm{years})$ & $72.48 \pm 4.65$ & $71.05 \pm 4.15$ & 2.854 & 0.005 \\
\hline $\mathrm{BMI}\left(\mathrm{kg} / \mathrm{m}^{2}\right)$ & $24.01 \pm 1.92$ & $25.20 \pm 2.31$ & -5.030 & 0.000 \\
\hline VFA $\left(\mathrm{cm}^{2}\right)$ & $98.03 \pm 13.11$ & $90.71 \pm 16.87$ & 4.438 & 0.000 \\
\hline Hypertension $(\%)$ & $28(31.46)$ & $105(29.09)$ & 0.193 & 0.660 \\
\hline Diabetes $(\%)$ & $14(15.73)$ & $49(13.57)$ & 0.276 & 0.599 \\
\hline Cardiac disease(\%) & $30(33.71)$ & $101(27.98)$ & 1.136 & 0.287 \\
\hline Smoking $(\%)$ & $15(16.85)$ & $49(13.57)$ & 0.630 & 0.427 \\
\hline Sports $(\%)$ & $10(11.24)$ & $75(20.78)$ & 4.241 & 0.039 \\
\hline $\mathrm{MNA}-\mathrm{SF}(\%)$ & $36(39.02)$ & $87(24.10)$ & 9.609 & 0.002 \\
\hline $\mathrm{TG}(\mathrm{mmol} / \mathrm{L})$ & $1.31 \pm 0.45$ & $1.51 \pm 0.58$ & -2.981 & 0.003 \\
\hline $\mathrm{LDL}-\mathrm{C}(\mathrm{mmol} / \mathrm{L})$ & $3.12 \pm 0.61$ & $3.08 \pm 0.62$ & 0.646 & 0.519 \\
\hline $\mathrm{HbA} 1 \mathrm{C}(\%)$ & $5.37 \pm 0.93$ & $5.28 \pm 1.12$ & 0.781 & 0.436 \\
\hline $\mathrm{Hb}(\mathrm{g} / \mathrm{L})$ & $125.73 \pm 11.97$ & $124.98 \pm 15.44$ & 0.426 & 0.670 \\
\hline $\mathrm{ALB}(\mathrm{g} / \mathrm{L})$ & $41.16 \pm 4.49$ & $44.19 \pm 4.43$ & -5.769 & 0.000 \\
\hline $\mathrm{Cr}(\mathrm{umol} / \mathrm{L})$ & $66.12 \pm 14.15$ & $72.37 \pm 11.15$ & -4.477 & 0.000 \\
\hline $\mathrm{baPWV}(\mathrm{cm} / \mathrm{s})$ & $1792.39 \pm 128.27$ & $1647.57 \pm 121.04$ & 9.989 & 0.000 \\
\hline $\mathrm{HS}(\mathrm{kg})$ & $23.99 \pm 5.60$ & $25.48 \pm 5.72$ & -2.216 & 0.027 \\
\hline $\mathrm{GS}(\mathrm{m} / \mathrm{s})$ & $0.86 \pm 0.26$ & $1.20 \pm 0.58$ & -5.433 & 0.000 \\
\hline $\mathrm{ASMI}\left(\mathrm{kg} / \mathrm{m}^{2}\right)$ & $6.59 \pm 0.73$ & $7.64 \pm 0.76$ & -11.738 & 0.000 \\
\hline
\end{tabular}

BMI: body mass index; ALB: serum albumin; TG: triacylglycerol; Cr: creatinine; VFA: visceral fat tissue; MNA-SF: mini-nutritional assessment short-form; BIA: Bioelectrical impedance analysis; ASMI: appendicular skeletal muscle index; Hb: hemoglobin; HbA1C: hemoglobin A1C; LDL-C: low-density lipoprotein cholesterol; baPWV: brachial ankle pulse wave velocity; HS: Handgrip strength; GS: usual gait speed.

Table 2 Multivariable linear regression analysis between baPWV and ASMI $\square$ muscle strength $\square$ physical performance

\begin{tabular}{lllll}
\hline Variable & $\beta$ & 95\%CI & $\mathrm{t}$ & $\mathrm{p}$ \\
\hline $\mathrm{ASMI}\left(\mathrm{kg} / \mathrm{m}^{2}\right)$ & -39.783 & -54.861 to -28.253 & -5.807 & 0.000 \\
\hline $\mathrm{HS}(\mathrm{kg})$ & -8.007 & -13.108 to -2.907 & -3.085 & 0.002 \\
\hline $\mathrm{GS}(\mathrm{m} / \mathrm{s})$ & -9.273 & -30.413 to 11.868 & -0.862 & 0.389 \\
\hline
\end{tabular}

* baPWV was taken as the dependent variable! ASMI, HS and GS were tested simultaneously as independent variables and models were adjusted for sex, age, BMI, VFA, hypertension, diabetes, cardiac, smoking, sports, MNA-SF, TG, LDL-C, HbA1C, Hb, ALB and Cr. 
BMI: body mass index; ALB: serum albumin; TG: triacylglycerol; Cr: creatinine; VFA: visceral fat tissue; MNA-SF: mini-nutritional assessment short-form; BIA: Bioelectrical impedance analysis; ASMI: appendicular skeletal muscle index; Hb: hemoglobin; HbA1C: hemoglobin A1C; LDL-C: low-density lipoprotein cholesterol; baPWV: brachial ankle pulse wave velocity; HS: Handgrip strength; GS: usual gait speed.

Table 3 Univariate regression analysis for correlative factor of sarcopenia

\begin{tabular}{|c|c|c|}
\hline Variable & OR (CI 95\%) & $\mathrm{p}$ \\
\hline Age(years) & 1.13ロ1.07-1.25凸 & 0.015 \\
\hline male/female $(0,1)$ & $0.76 \square 0.65-1.23 \square$ & 0.375 \\
\hline $\operatorname{BMI}\left(\mathrm{kg} / \mathrm{m}^{2}\right)$ & $0.78 \square 0.66-0.92 \square$ & 0.036 \\
\hline Hypertension $(0,1)$ & 1.26ロ0.87-1.49! & 0.052 \\
\hline Diabetes $(0,1)$ & 1.58ロ0.92-1.87ロ & 0.129 \\
\hline Smoking $(0,1)$ & 1.07ロ0.86-1.27凸 & 0.238 \\
\hline Cardiac disease $(0,1)$ & $0.89 \square 0.90-1.15 \square$ & 0.284 \\
\hline Sports $(0,1)$ & $0.80 \square 0.66-0.93 \square$ & 0.042 \\
\hline MNA-SF $(0,1)$ & 0.83ロ0.75-0.94ロ & 0.038 \\
\hline $\mathrm{TG}(\mathrm{mmol} / \mathrm{L})$ & $0.88 \square 0.76-1.08 \square$ & 0.687 \\
\hline LDL-C(mmol/L) & $0.81 \square 0.47-1.07 \square$ & 0.531 \\
\hline HbA1C $(\%)$ & 0.92凸0.81-1.07ロ & 0.512 \\
\hline $\mathrm{Hb}(\mathrm{g} / \mathrm{L})$ & $0.68 \square 0.61-1.17 \square$ & 0.074 \\
\hline $\mathrm{ALB}(\mathrm{g} / \mathrm{L})$ & $0.85 \square 0.76-1.12 \square$ & 0.052 \\
\hline $\mathrm{Cr}(\mathrm{umol} / \mathrm{L})$ & $0.86 \square 0.70-1.25 \square$ & 0.063 \\
\hline $\operatorname{VFA}\left(\mathrm{cm}^{2}\right)$ & $0.59 \square 0.48-0.83 \square$ & 0.041 \\
\hline $\mathrm{BaPWV}(\mathrm{cm} / \mathrm{s})$ & 1.28ロ1.07-1.46ロ & 0.027 \\
\hline
\end{tabular}

OR: odds ratio, CI: confidence interval, BMI: body mass index; ALB: serum albumin; TG: triacylglycerol; Cr: creatinine; VFA: visceral fat tissue; MNA-SF: mini-nutritional assessment short-form; BIA: Bioelectrical impedance analysis; ASMI: appendicular skeletal muscle index; Hb: hemoglobin; HbA1C: hemoglobin A1C; LDL-C: low-density lipoprotein cholesterol; baPWV: brachial ankle pulse wave velocity; HS: Handgrip strength; GS: usual gait speed.

Table 4 Multiple logistic regression analysis for correlative factor of sarcopenia

\begin{tabular}{lcc}
\hline Variable & OR (CI 95\%) & $\mathrm{p}$ \\
\hline Age(years) & $1.21 \square 1.07-1.32 \square$ & 0.026 \\
\hline BMI $\left(\mathrm{kg} / \mathrm{m}^{2}\right)$ & $0.74 \square 0.65-0.86 \square$ & 0.035 \\
\hline Sports $(0,1)$ & $0.68 \square 0.53-0.79 \square$ & 0.046 \\
\hline MNA-SF $(0,1)$ & $1.08 \square 0.81-1.24 \square$ & 0.652 \\
\hline VFA $\left(\mathrm{cm}^{2}\right)$ & $0.65 \square 0.52-1.03 \square$ & 0.271 \\
\hline BaPWV(cm/s) & $1.68 \square 1.45-1.87 \square$ & 0.037 \\
\hline
\end{tabular}

OR: odds ratio, CI: confidence interval, BMI: body mass index; VFA: visceral fat tissue; baPWV: brachial ankle pulse wave velocity; MNA-SF: mini-nutritional assessment short-form. 